\title{
BMJ Open Study protocol of a population-based cohort investigating Physical Activity, Sedentarism, lifestyles and Obesity in Spanish youth: the PASOS study
}

Santiago Felipe Gómez (D) ,1,2 Clara Homs, ${ }^{1,3}$ Julia Wärnberg, ${ }^{4,5}$ Maria Medrano, ${ }^{6}$ Marcela Gonzalez-Gross, ${ }^{5,7}$ Narcis Gusi, ${ }^{8}$ Susana Aznar, ${ }^{9}$ Elena Marín Cascales, ${ }^{10}$ Miguel González-Valeiro, ${ }^{11}$ Lluis Serra-Majem, ${ }^{12,13}$ Nicolás Terrados, ${ }^{14}$ Josep A Tur, ${ }^{15}$ Marta Segú, ${ }^{16}$ Camille Lassale (D),${ }^{17,18}$ Juan Carlos Benavente-Marín, ${ }^{4}$ Idoia Labayen, ${ }^{6}$ 'Augusto García Zapico, ${ }^{7,19}$ Jesús Sánchez-Gómez, ${ }^{8}$ Fabio Jiménez-Zazo, ${ }^{9}$ Pedro Emilio Alcaraz, ${ }^{10,20}$ Marta Sevilla-Sanchez (D) , ${ }^{11}$ Estefania Herrera-Ramos, ${ }^{12}$ Susana Pulgar, ${ }^{14}$ Maria del Mar Bibiloni, ${ }^{5,15}$ Olga Sancho, ${ }^{16}$ Helmut Schröder ${ }^{17,18}$

To cite: Gómez SF, Homs C, Wärnberg J, et al. Study protocol of a population-based cohort investigating Physical Activity, Sedentarism, lifestyles and Obesity in Spanish youth: the PASOS study. BMJ Open 2020;10:e036210. doi:10.1136/ bmjopen-2019-036210

- Prepublication history and additional material for this paper are available online. To view these files, please visit the journal online (http://dx.doi. org/10.1136/bmjopen-2019036210).

Received 10 December 2019 Revised 16 July 2020 Accepted 17 August 2020

Check for updates

(c) Author(s) (or their employer(s)) 2020. Re-use permitted under CC BY-NC. No commercial re-use. See rights and permissions. Published by BMJ.

For numbered affiliations see end of article.

Correspondence to Dr Santiago Felipe Gómez; sgomez@gasolfoundation.org

\section{ABSTRACT}

Introduction Physical activity (PA) is essential to healthy mental and physical development in early life. However, the prevalence of physical inactivity, which is considered a key modifiable driver of childhood obesity, has reached alarming levels among European youth. There is a need to update the data for Spain, in order to establish if current measures are effective or new approaches are needed. Methods and analysis We present the protocol for Physical Activity, Sedentarism, lifestyles and Obesity in Spanish youth (PASOS). This observational, nationally representative, multicentre study aims to determine the PA levels, sedentary behaviours and prevalence of physical inactivity (defined as $<60$ min of moderate to vigorous PA per day) in a representative sample of Spanish children and adolescents. The PASOS study has recruited a representative random sample of children and adolescents aged 8-16 years from 242 educational centres in the 17 'autonomous regions' into which Spain is divided. The aim is to include a total of 4508 youth participants and their families. Weight, height and waist circumference will be measured by standardised procedures. Adherence to the Mediterranean diet, quality of life, sleep duration, PA and sedentary behaviour are being measured by validated questionnaires. PA is measured by the Physical Activity Unit 7-item Screener. A representative subsample ( $10 \%$ of participants) was randomly selected to wear accelerometers for 9 days to obtain objective data on PA. Parents are asked about their educational level, time spent doing PA, diet quality, self-perceived stress, smoking habit, weight, height, their child's birth weight and if the child was breast fed.

Ethics and dissemination The study was approved by the Ethics Committee of the Fundació Sant Joan de Déu, Barcelona, Spain. Main findings of the study will be disseminated to the scientific community and to general public by media conferences, social media and a website. Trial registration number ISRCTN34251612.

\section{Strengths and limitations of this study}

- The Physical Activity, Sedentarism, lifestyles and Obesity in Spanish youth study is a representative nationwide survey among Spanish youth.

- It provides data about physical activity, lifestyles and weight status.

- Sociodemographic data and parents' lifestyle variables are also evaluated.

- The study is not designed to provide representative data for each region.

\section{INTRODUCTION}

Physical inactivity is one of the leading risk factors for premature death worldwide, ${ }^{1}$ putting an enormous economic burden on the public health system. ${ }^{2}$ The 2018 Physical Activity Guidelines for Americans underlined the paramount importance of this modifiable health behaviour in children and adults of all ages. ${ }^{3}$ Physical inactivity is associated with an increased risk of overweight and obesity in children and adolescents. ${ }^{4}$ Therefore, the high level of insufficient physical activity (PA), particularly in western countries, ${ }^{5}$ is of great concern. Perhaps more alarming was a 2012 report $^{6}$ because it indicated that $80.3 \%$ of adolescents worldwide did not meet the minimum $60 \mathrm{~min}$ of moderate to vigorous PA (MVPA) per day recommended by the $\mathrm{WHO}^{1}$ for children and adolescents. Objectively measured PA data from the Healthy Lifestyle in Europe by Nutrition in Adolescence study showed that over half of the boys and nearly one-third of the girls met the PA 
recommendations, but spent on average $70 \%$ of their waking time in sedentary behaviours. ${ }^{7}$ The Identification and Prevention of Dietary and Lifestyle-induced Health Effects in Children and Infants study reported similar data for Europe. $^{8}$ Results from a recently published review on objectively measured $\mathrm{PA}^{9}$ revealed that $71 \%$ of European children and adolescents were physically active less than 1 hour/day and with considerable variability between countries. Furthermore, PA level decreased from north to south Europe. ${ }^{9}$ Self-reported data from the Health Behaviour in School-aged Children (HBSC) study on secular trends of European adolescents not meeting the PA recommendation showed mixed results across countries. ${ }^{10}$

Most large epidemiological studies, such as HBSC, use questionnaires because self-reported data collection by validated questionnaires is cost-effective and therefore feasible to implement. However, self-reporting presents inherent limitations, as it is prone to response and classification bias, and therefore to measurement error. ${ }^{11}$ Therefore, the Report on Physical Activity for Spanish Children and Adolescents ${ }^{12}{ }^{13}$ underlined the need to obtain objectively measured data in order to better estimate the number of Spanish youths not meeting the PA recommendations. Objective PA measurement can also be used to calibrate self-reported PA data, when both types of data are collected in the same individuals. This can reduce measurement error in questionnaire-derived PA estimates.

Furthermore, the identification and understanding of PA correlates and determinants is essential for the creation and implementation of intervention programmes aiming to increase PA in children and adolescents. Associations between PA and demographic, socioeconomic, psychological, social and behavioural factors have been reported in children, but the evidence is inconclusive. ${ }^{14-17}$

This manuscript describes the rationale and design of the Physical Activity, Sedentarism, lifestyles and Obesity in Spanish youth (PASOS) study, which aims to determine PA levels and its correlates in Spanish children and adolescents.

\section{Objectives}

Main objective

To determine the PA levels, sedentary behaviours and prevalence of physical inactivity in a representative sample of Spanish children and adolescents.

\section{Secondary objectives}

1. To determine the prevalence of general and abdominal obesity.

2. To calibrate the self-reported Physical Activity Unit 7item Screener (PAU-7S) using objective accelerometer data from a representative subsample of $10 \%$ of the cohort.

3. To examine the inter-relationships between PA and demographic, lifestyle, socioeconomic, behavioural, anthropometric and environmental factors.
Incidences of obesity and physical inactivity in addition with a prospective data analysis of objective 3 will be addressed with follow-up data at the end of 2022.

\section{METHODS/DESIGN \\ Study design}

This is a multicentre, cross-sectional, nationally representative, population-based study. A follow-up of the study participants is planned for 2022 with a repeated collection of all baseline data.

\section{Inclusion criteria}

Children and adolescents aged 8-16 years who were enrolled in a participating school were eligible for inclusion.

\section{Exclusion criteria}

Individuals with an intellectual disability that prevents response to the lifestyle questionnaires were excluded of the baseline data collection. Each case was evaluated with the corresponding teachers and parents or legal guardians before exclusion.

\section{Randomisation}

Randomisation was performed by a multistage sampling procedure $^{1819}$ including four stages. To obtain a sample of 4508 children/adolescents, assuming a mean of 18-20 pupils per classroom, 242 participating classrooms were required from the 17 'autonomous communities' into which Spain is divided: 121 from primary schools (grades 3-6) and 121 from secondary schools (levels 1-4). In the first step, 121 municipalities were randomised across three population strata: 2000-30 000; 30001-200000; and more than 200000 inhabitants. The total number of selected municipalities in each autonomous community was proportional to its share of the youth population of Spain aged 8-16 years. ${ }^{20}$ In a second step, 242 schools were randomised from the selected municipalities, along with up to three replacements for each selected school to account for census data error or centres not willing to participate. In a third step, scholar-year per school was randomised. In the fourth and final step, a classroom for each scholar-year was randomised and invited to participate. A subsample of 23 classrooms $(10 \%)$ was randomised for the objective measurement of PA by accelerometers. The software used for the sampling procedure were R, package mstage.

\section{Sample size}

The calculation of the sample size was based on the prevalence of non-adherence to PA recommendations of at least 1 hour of PA per day. ${ }^{21}$ According to the published data of the Spanish Report of Physical Activity, ${ }^{12}{ }^{13}$ we assumed 50\% non-adherence among Spanish children and adolescents. Based on this assumption and considering a population increase of $8 \%$ as a relevant indicator, a total of 3994 participants were needed, 1997 in each of the two age groups (primary school: 8-11 years, and 
secondary school: 12-16 years), to achieve a statistical power of $\geq 80 \%$ to identify an increase of $8 \%$ as significant $(\mathrm{p} \leq 0.05)$. A dropout rate of $20 \%$ was anticipated. To take into account the cluster effect, sample size was increased by $10 \%$, leading to a sample size of 4394 participants. Finally, to ensure proportionality among the 17 autonomous communities studied, the number of municipalities was increased to 121 and the final sample to 4508 participants.

\section{Data collection}

Baseline data were collected from March 2019 to February 2020, in 242 primary and secondary schools. Two visits were carried out in each school by two field researchers with a background in physical education (PE), nutrition or other health sciences. They completed a 1-day training session on the project methodology, hosted by the Gasol Foundation. An additional visit was made to the $10 \%$ of the schools included in the accelerometer protocol. Lifestyle data of children/adolescents were self-reported online at participating schools, with the assistance of trained personnel. Parental sociodemographic and lifestyle (PA and smoking) data were collected in paper format. Additional data on parental health habits were recorded via an online system.

\section{Participants and recruitment process}

The aim was to recruit 4508 children and their parents from 242 participating schools in the 17 autonomous communities. Ceuta and Melilla, two autonomous cities in North Africa with less than $0.8 \%$ of the total Spanish population aged 8-16 years, were not included for logistical reasons.

This cohort study is coordinated by the Gasol Foundation, whose aim is to reduce childhood obesity rates through the promotion of sports and PA, healthy eating, sleep quality and the emotional well-being of children, adolescents and their families in the USA and Spain. Field and scientific work is being performed together with 13 highly experienced research groups working at universities and research centres in several regions of Spain (online supplemental file 1). Selected educational centres first received an invitation letter signed by the president of the Gasol Foundation (Pau Gasol) and accompanied by support letters from the autonomous community's departments of education and/or health and sports and from Spain's Ministry of Education and Vocational Training; Ministry of Health, Consumer Affairs and Social Welfare; Council of Sports and High Commission against Child Poverty. In a second step, Gasol Foundation staff called the invited educational centres to confirm their interest in participating. The principal investigators at these centres are well-known experts in the investigation of the relationship between lifestyle and disease. In a third step, the regional coordinators of the PASOS project contacted the interested educational centres to introduce them to the study and invited them to participate. In the participating schools, parents (or legal guardians) were contacted by teachers designated by school administrators and received an envelope containing instructions to complete the requested documentation, two copies of the informed consent form, and two copies of the short questionnaires to be completed by an adult. When the school received a signed copy of the informed consent form, the child participant and family were included in the PASOS study. The study was approved by the Ethics Committee of the Fundació Sant Joan de Déu, Barcelona, Spain.

\section{Study variables}

\section{Children and adolescents}

All the children and adolescent variables were gathered during school hours, using an online system for questionnaires and with the logistical help of teachers to organise evaluation sessions.

\section{Physical activity}

The PAU-7S, a 7-item self-reported questionnaire, was used to assess PA levels in each participating child or adolescent.

Six questions ask about PA frequency and duration in the previous week: (1) How many days did you go for a walk? (2) How many days did you participate in movement play during recess time? (3) How many days did you participate in movement play during free time after school or during the weekend? (4) How many days did you have PE class at school? (5) How many days did you play a team sport? (6) How many days did you play an individual sport? The response options for these questions about PA are shown in a table with a box for each day of the week, in which children can mark if they have spent: (1) 0 min (no activity); (2) less than $30 \mathrm{~min}$; (3) between $30 \mathrm{~min}$ and 1 hour; (4) between 1 hour and 1.5 hours; or (5) more than 1.5 hours. The final question asks about health status with a Yes/No response option: Were you sick last week or did anything prevent you from performing your usual PA?

In addition, PA was objectively measured by accelerometers in $10 \%$ of the participants, randomly selected from the entire sample. For 9 days, these children wore the ActiGraph wGT3X-BT (Pensacola, FL, USA) accelerometer. Total PA, PA intensity, sedentary time and sleep duration were recorded. Furthermore, children reported non-wear time, bedtime and wake time in a daily log. Trained personnel instructed them how to report this information.

\section{Anthropometric variables}

Anthropometrics for each individual were measured by trained personnel following the WHO standardised protocol. ${ }^{22}$ Body weight, height and waist circumference were measured with the children in light clothing, without shoes. The measurements were performed using an electronic SECA 899 scale (recorded to the nearest $100 \mathrm{~g}$ ), a portable SECA 217 stadiometer (to the nearest 1 $\mathrm{mm}$ ) and a flexible, non-stretch SECA 201 metric tape (to the nearest $1 \mathrm{~mm}$ ), respectively. Waist circumference was 
measured in the narrowest zone between the lower costal rib and iliac crest, in the supine decubitus and horizontal positions.

\section{Other child/adolescent lifestyle variables}

Sedentary behaviour was assessed by the Screen-time Sedentary Behaviour Questionnaire, ${ }^{23}$ which asks about time spent in four activities: (1) watching TV, (2) playing computer games, (3) playing console (video) games, and (4) using a mobile phone, separately for weekdays and weekends.

Diet was assessed by the 16-item KIDMED questionnaire(Kids level of adherence to the Mediterranean diet). ${ }^{24}$ The KIDMED index, derived on the basis of dichotomous response options (Yes/No), was created to estimate adherence to the Mediterranean diet in children and young adults, based on the principles that sustain Mediterranean dietary patterns and those that undermine it. Items denoting lower adherence are assigned a value of -1 ( 4 items) and those related to higher adherence are scored +1 (12 items).

Sleep duration was recorded by four questions on hours of sleep from the Sleep Habits Survey for Adolescents that ask about bedtime and time of waking up on weekdays and weekends. ${ }^{25}$

In addition, adults were asked to complete the BEARS (Bedtime, Excessive, Awakening, Regularity, Snoring) questionnaire on sleep quality in their participating children/adolescents, ${ }^{26}$ responding (Yes/No) to questions about five main sleep domains: $\mathrm{A}=$ bedtime problems, $\mathrm{B}=$ excessive daytime sleepiness, $\mathrm{C}=$ awakening during the night, $\mathrm{D}=$ regularity and duration of sleep, $\mathrm{E}=$ snoring.

Quality of life was measured by the 'EQ-5D-Y-5L'-a short, child-friendly EuroQuality questionnaire on five health-related dimensions (mobility, self-care, usual activities, pain/discomfort, anxiety/depression) with five response levels, recently validated in an international sample (including Spain) of children and adolescents aged $8-15$ years. ${ }^{27}$ To facilitate comparison with other studies, the 3-level version (EQ-5D-Y-3L) was also to be administered. ${ }^{28}$

\section{Parental variables}

Two sets of questionnaires were delivered to each participating child/adolescent, to be answered separately by up to two parents/legal guardians. The validated REGICOR (REgistre GIroní del COR) short PA Questionnaire ${ }^{29}$ and the following standardised questions were included: sex, weight, height, smoking habit, educational level, employment status, general health status and sleep duration. Parents were also asked about their child's birth weight and if the child was breast fed.

Additionally, parents/legal guardians were asked to respond to the following online questionnaires:

- Diet Quality Screener, a short questionnaire about the frequency of consumption of 18 foods/food groups. ${ }^{30} 31$
- Perceived Stress Scale, a subjective 14-item questionnaire asking about self-perception of stress experienced during the previous month. ${ }^{32}$

- Environmental questions such as access to cycling lanes or sports facilities are asked to capture information that can be used to estimate the level of exposure to contaminants such as air pollution and noise levels or access to green spaces.

- Quality of life, measured by adult response to the child-friendly EQ-5D-Y-5L. ${ }^{27}$

Parental variables will be used to study the crosssectional and prospective association between parental lifestyle, such as PA and diet quality, with the corresponding child lifestyle.

\section{Statistical analysis}

A descriptive analysis of the data and a depuration of the database will be carried out to minimise errors. Before the construction of statistical models, quantitative variables will be checked according to their distribution, and, if necessary, logarithmic transformation will be carried out. Multivariate logistic and linear regression models will be carried out to determine the associations of exposures and outcomes of interest in cross-sectional and prospective analysis. Additionally, general linear models with post hoc Bonferroni correction for repeated measurements will be executed. To address specific research questions, appropriate statistical models, such as principal component analysis, cluster analysis and mediation analysis, will be applied. Linear and logistic regression models with cubic spline functions will be fitted to determine the dose-response relationship between exposure and outcome.

\section{Patient and public involvement}

Patients and the public were not involved in the development of the research question or in the design of the study. The parents and legal tutors of participant children received the informed consent and a letter inviting them to participate in the study. Also, parents and legal tutors received a one-page plain language summary of the results of the anthropometric measurements. The baseline results of the PASOS study are being disseminated to the general public, an activity that will continue during the cohort study.

\section{DISCUSSION}

The PASOS study is intended to provide an estimation of PA among Spanish children and adolescents and, conversely, the magnitude of physical inactivity and sedentarism. The representative design of the study is of particular importance because estimates of physical inactivity can vary widely by population. ${ }^{33}$ Moreover, data obtained by accelerometers in a representative subsample of $10 \%$ of the entire cohort will be used to calibrate self-reported PA data obtained from the PAU-7S and to provide an objective and representative measure of PA levels and of 
the prevalence of physical inactivity in Spanish children and adolescents.

Some technical considerations must be kept in mind. First, accelerometer-derived calculation of time spent in MVPA is based on the count-per-minute threshold for MVPA that is applied. ${ }^{34-36}$ Differences in this parameter can strongly affect the estimate of PA and the classification of adherence to PA guidelines. ${ }^{34}{ }^{35}$ For example, Gába and colleagues ${ }^{34}$ showed that adherence to the PA guidelines ranged from $1 \%$ to $100 \%$ depending on the algorithm used. Therefore, the comparability of accelerometer-based PA measurements is limited across studies.

To improve comparability of accelerometer-driven MVPA data from the PASOS study with other research, several different accelerometer MVPA cut-off points and epoch lengths used in other studies will be analysed and made public. In addition, calibration of the PAU-7S will be stratified by sex and age group (primary vs secondary school students).

The association between physical inactivity and increased risk of overweight and obesity in children and adolescents $^{4}$ has multiple potential consequences ${ }^{37}$ that persist into adulthood. In addition, psychosocial restrictions such as impaired quality of life, self-esteem and school performance are to be expected. ${ }^{38} \mathrm{~A}$ recent metaanalysis showed that children aged 5-15 years who are overweight or obese have more cardiometabolic complications than children of normal weight. ${ }^{39}$ Furthermore, a cohort study found that some risk factors, including fatty liver, are detectable even in overweight or obese preschoolers.

Spain is among the European countries with the highest prevalence of overweight, obesity and severe obesity in children and adolescents. ${ }^{40}$ However, representative data on the nationwide prevalence of abdominal obesity in Spanish children and adolescents aged 8-16 years have not been published since $2000 .{ }^{42}$ Results of the PASOS study will provide timely data on PA behaviours, the prevalence of overweight and obesity, and estimated secular trends in abdominal obesity in a representative sample of this population. Finally, our analysis of potential determinants of PA behaviours will improve our understanding of which of these determinants are modifiable and accessible for intervention. Our findings will contribute essential knowledge for the development and implementation of effective PA promotion strategies in multilevel intervention programmes designed to tackle childhood obesity.

\footnotetext{
Author affiliations

${ }^{1}$ Programs, Gasol Foundation, Sant Boi de Llobregat, Barcelona, Spain

${ }^{2}$ GREpS, Health Education Research Group, Nursing and Physiotherapy Department, University of Lleida, Lleida, Catalunya, Spain

${ }^{3}$ Global Research on Wellbeing (GRoW), Blanquerna Ramon Llull University Faculty

of Health Sciences, Barcelona, Catalunya, Spain

${ }^{4}$ Faculty of Health Sciences, Institute of Biomedical Research of Malaga (IBIMA), University of Málaga, Malaga, Andalucía, Spain

${ }^{5}$ Centro de Investigación Biomédica en Red-Fisiopatología de la Obesidad y la Nutrición (CIBEROBN), Carlos III Health Institute, Madrid, Spain
}

${ }^{6}$ ELIKOS Group, Institute for Innovation and Sustainable Development in Food Chain (IS-F00D), Public University of Navarre, Pamplona, Navarra, Spain ${ }^{7}$ ImFINE Research Group, Department of Health and Human Performance, Universidad Politecnica de Madrid, Madrid, Comunidad de Madrid, Spain ${ }^{8}$ Physical Activity and Quality of Life Research Group (AFYCAV), Faculty of Sport Sciences, University of Extremadura, Caceres, Extremadura, Spain

${ }^{9}$ PAFS Research Group, Faculty of Sports Sciences, University of Castilla-La Mancha-Toledo Campus, Toledo, Castilla-La Mancha, Spain

${ }^{10}$ Research Center for High Performance Sport, San Antonio Catholic University of Murcia, Murcia, Spain

${ }^{11}$ Faculty of Sports Sciences and Physical Education, Universidade da Coruña, A Coruna, Galicia, Spain

${ }^{12}$ Research Institute of Biomedical and Health Sciences (IUIBS), University of Las Palmas de Gran Canaria, Las Palmas, Canary Islands, Spain

${ }^{13}$ Preventive Medicine Service, Centro Hospitalario Universitario Insular Materno Infantil (CHUIMI), Canarian Health Service, Las Palmas, Spain

${ }^{14}$ Regional Unit of Sports Medicine, Municipal Sports Foundation of Avilés, Avilés, Spain

${ }^{15}$ Research Group of Community Nutrition and Oxidative Stress, University of the Balearic Islands, Palma de Mallorca, Illes Balears, Spain

${ }^{16}$ Probitas Foundation, Barcelona, Spain

${ }^{17}$ CIBER Epidemiology and Public Health (CIBERESP), Carlos III Health Institute, Madrid, Spain

${ }^{18}$ Cardiovascular Risk and Nutrition Research Group, Hospital del Mar Institute for Medical Research, Barcelona, Catalunya, Spain

${ }^{19}$ Department of Didactics of Language, Arts and Physical Education, Universidad Complutense de Madrid, Madrid, Comunidad de Madrid, Spain

${ }^{20}$ Faculty of Sport Sciences, San Antonio Catholic University of Murcia, Murcia, Spain

Twitter Santiago Felipe Gómez @SantiGomez_SF and Augusto García Zapico @ agz15

Acknowledgements We thank the staff, pupils, parents, schools and municipalities for their participation, enthusiasm and support. We appreciate the English revision by Elaine M Lilly, PhD.

Contributors SFG, CH and HS conceptualised and designed the study, drafted the initial manuscript and incorporated the suggestions of all the consortium authors. JW, JCBM, MM, SA, FJZ, MM and IL made relevant contributions to the accelerometry protocol and formed with SFG, CH and HS the accelerometry commission of the PASOS study. MGG, NG and JAT made substantial contributions to the study protocol and the representative randomisation of the Spanish population. JW, MM, MGG, SA, IL and AGZ made a relevant intellectual input to the manuscript content. SFG, CH, JW, MM, MGG, NG, SA, EMC, MGV, LSM, NT, JAT, MS, CL, JCBM, IL, AGZ, JSG, FJZ, PEA, MSS, EHR, SP, MdMB, OS and HS defined the strategy to deploy the study protocol in their assigned schools and reviewed, contributed and approved the final manuscript as submitted.

Funding PASOS study has been funded mainly by Fundación PROBITAS and Gasol Foundation. Additional funds were received from the Barça Foundation, Banco Santander, IFA, Viena and Fundación Deporte Jóven. MMB is funded by the official funding agency for biomedical research of the Spanish government, Institute of Health Carlos III (ISCIII), which is cofunded by the European Regional Development Fund (CIBEROBN CB12/03/30038), grant of support to research groups number 35/2011 (Balearic Islands Gov) and EU COST Action CA16112. PASOS has the institutional support of Spain's Ministry of Education and Vocational Training, the Ministry of Health, Consumption and Social Welfare through the Spanish Agency for Food Safety and Nutrition (ASEAN), the High Commission against Child Poverty, the High Sports Council, the General College of Professional Associations of Physical Education and Sports, and the Departments of Education and/or Health and/or Sports of Spain's 17 autonomous regions. The CIBERESP and the CIBEROBN are initiatives of the Institute of Health Carlos III, Madrid, Spain.

Competing interests None declared.

Patient and public involvement Patients and/or the public were involved in the design, or conduct, or reporting, or dissemination plans of this research. Refer to the Methods section for further details.

Patient consent for publication Not required.

Ethics approval Ethical approval was obtained from the Ethics Committee of the Fundació Sant Joan de Déu, Barcelona, Spain. Findings will be disseminated in seminars, conference presentations and in peer-reviewed international journals. 
Provenance and peer review Not commissioned; externally peer reviewed.

Open access This is an open access article distributed in accordance with the Creative Commons Attribution Non Commercial (CC BY-NC 4.0) license, which permits others to distribute, remix, adapt, build upon this work non-commercially, and license their derivative works on different terms, provided the original work is properly cited, appropriate credit is given, any changes made indicated, and the use is non-commercial. See: http://creativecommons.org/licenses/by-nc/4.0/.

\section{ORCID iDs}

Santiago Felipe Gómez http://orcid.org/0000-0003-3352-2510

Camille Lassale http://orcid.org/0000-0002-9340-2708

Marta Sevilla-Sanchez http://orcid.org/0000-0003-2004-9162

\section{REFERENCES}

1 WHO Fact sheets. Physical activity 2018. Available: https://www. who.int/news-room/fact-sheets/detail/physical-activity

2 Ding D, Lawson KD, Kolbe-Alexander TL, et al. The economic burden of physical inactivity: a global analysis of major non-communicable diseases. Lancet 2016;388:1311-24.

3 Piercy KL, Troiano RP, Ballard RM, et al. The physical activity guidelines for Americans. JAMA 2018;320:2020-8.

4 Hills AP, Andersen LB, Byrne NM. Physical activity and obesity in children. Br J Sports Med 2011;45:866-70.

5 Guthold R, Stevens GA, Riley LM, et al. Worldwide trends in insufficient physical activity from 2001 to 2016: a pooled analysis of 358 population-based surveys with 1.9 million participants. Lancet Glob Health 2018;6:e1077-86.

6 Hallal PC, Andersen LB, Bull FC, et al. Global physical activity levels: surveillance progress, pitfalls, and prospects. Lancet 2012;380:247-57.

7 Ruiz JR, Ortega FB, Martínez-Gómez D, et al. Objectively measured physical activity and sedentary time in European adolescents: the Helena study. Am J Epidemiol 2011;174:173-84.

8 Konstabel K, Veidebaum T, Verbestel V, et al. Objectively measured physical activity in European children: the IDEFICS study. Int $J$ Obes 2014;38 Suppl 2:S135-43.

9 Steene-Johannessen J, Hansen BH, Dalene KE, et al. Variations in accelerometry measured physical activity and sedentary time across Europe - harmonized analyses of 47,497 children and adolescents. Int J Behav Nutr Phys Act 2020;17:38.

10 Kalman M, Inchley J, Sigmundova D, et al. Secular trends in moderate-to-vigorous physical activity in 32 countries from 2002 to 2010: a cross-national perspective. Eur J Public Health 2015;25 Suppl 2:37-40.

11 Sirard JR, Pate RR. Physical activity assessment in children and adolescents. Sports Med 2001;31:439-54.

12 Informe 2016: Actividad Física en niños Y adolescentes en España. Fundación para La Investigación Nutricional. Available: https://www. activehealthykids.org/wp-content/uploads/2016/11/spain-reportcard-long-form-2016.pdf

13 Roman-Viñas B, Marin J, Sánchez-López M, et al. Results from Spain's 2016 report card on physical activity for children and youth. $J$ Phys Act Health 2016;13:S279-83.

14 Craggs C, Corder K, van Sluijs EMF, et al. Determinants of change in physical activity in children and adolescents: a systematic review. Am J Prev Med 2011;40:645-58.

15 O'Donoghue G, Kennedy A, Puggina A, et al. Socio-economic determinants of physical activity across the life course: A "DEterminants of Dlet and Physical ACtivity" (DEDIPAC) umbrella literature review. PLoS One 2018;13:e0190737.

16 Condello G, Puggina A, Aleksovska K, et al. Behavioral determinants of physical activity across the life course: a "DEterminants of Dlet and Physical ACtivity" (DEDIPAC) umbrella systematic literature review. Int J Behav Nutr Phys Act 2017;14:58.

17 Cortis C, Puggina A, Pesce C, et al. Psychological determinants of physical activity across the life course: A "DEterminants of Dlet and Physical ACtivity" (DEDIPAC) umbrella systematic literature review. PLoS One 2017;12:e0182709.

18 Steel D. Multistage Sampling. In: Lovric M, ed. International encyclopedia of statistical science. Berlin, Heidelberg: Springer, 2011.
19 Sedgwick P. Multistage sampling. BMJ 2015;351:h4155.

20 Instituto Nacional de Estadística 2018. Available: http://www.ine.es/ jaxi/Tabla.htm?path=/t20/e245/p08/l0/\&file=02002.px

21 WHO - World Health Organization. Recommended levels of physical activity for children aged 5-17 years. global recommendations on physical activity for health. Available: http://www.who.int/ dietphysicalactivity/factsheet_young_people/en/

22 WHO - World Health Organization. Weighing and Measuring a Child. In: Training course and other tools, 2019. https://www.who.int/ childgrowth/training/en/

23 Rey-López JP, Ruiz JR, Ortega FB, et al. Reliability and validity of a screen time-based sedentary behaviour questionnaire for adolescents: the Helena study. Eur J Public Health 2012;22:373-7.

24 Serra-Majem L, Ribas L, Ngo J, et al. Food, youth and the Mediterranean diet in Spain. development of KIDMED, Mediterranean diet quality index in children and adolescents. Public Health Nutr 2004;7:931-5.

25 Wolfson AR, Carskadon MA, Acebo C, et al. Evidence for the validity of a sleep habits survey for adolescents. Sleep 2003;26:213-6.

26 Owens JA, Dalzell V. Use of the 'BEARS' sleep screening tool in a pediatric residents' continuity clinic: a pilot study. Sleep Med 2005;6:63-9.

27 Kreimeier S, Åström M, Burström K, et al. EQ-5D-Y-5L: developing a revised EQ-5D-Y with increased response categories. Qual Life Res 2019;28:1951-61.

28 EuroQol Group. EuroQol--a new facility for the measurement of health-related quality of life. Health Policy 1990;16:199-208.

29 Molina L, Sarmiento M, Peñafiel J, et al. Validation of the REGICOR short physical activity questionnaire for the adult population. PLOS One 2017;12:e0168148.

30 Schröder H, Benitez Arciniega A, Soler C, et al. Regicor Investigators; Hermes Investigators. validity of two short screeners for diet quality in time-limited settings. Public Health Nutr 2012;15:618-26.

31 Funtikova A, Baena-Díez JM, Koebnick C, et al. Validity of a short diet-quality index to predict changes in anthropometric and cardiovascular risk factors: a simulation study. Eur J Clin Nutr 2012;66:1369-71.

32 Cohen S, Kamarck T, Mermelstein R. A global measure of perceived stress. J Health Soc Behav 1983;24:385-96.

33 Guinhouya BC, Samouda H, de Beaufort C. Level of physical activity among children and adolescents in Europe: a review of physical activity assessed objectively by accelerometry. Public Health 2013;127:301-11.

34 Gába A, Dygrýn J, Mitáš J, et al. Effect of Accelerometer cut-off points on the recommended level of physical activity for obesity prevention in children. PLoS One 2016;11:e0164282.

35 Brazendale K, Beets MW, Rowlands AV, et al. Converting between estimates of moderate-to-vigorous physical activity derived from raw accelerations measured at the wrist and from ActiGraph counts measured at the hip: the Rosetta stone. J Sports Sci 2018;36:2603-7.

36 Banda JA, Haydel KF, Davila T, et al. Effects of varying epoch lengths, wear time algorithms, and activity Cut-Points on estimates of child sedentary behavior and physical activity from Accelerometer data. PLoS One 2016;11:e0150534.

37 Whooten R, Kerem L, Stanley T. Physical activity in adolescents and children and relationship to metabolic health. Curr Opin Endocrinol Diabetes Obes 2019;26:25-31.

38 de Greeff JW, Bosker RJ, Oosterlaan J, et al. Effects of physical activity on executive functions, attention and academic performance in preadolescent children: a meta-analysis. J Sci Med Sport 2018;21:501-7.

39 Friedemann C, Heneghan C, Mahtani K, et al. Cardiovascular disease risk in healthy children and its association with body mass index: systematic review and meta-analysis. BMJ 2012;345:e4759.

40 Sánchez-Cruz J-J, Jiménez-Moleón JJ, Fernández-Quesada F, et al. Prevalence of child and youth obesity in Spain in 2012. Rev Esp Cardiol 2013;66:371-6.

41 Cadenas-Sanchez C, Intemann T, Labayen I, et al. Prevalence of severe/morbid obesity and other weight status and anthropometric reference standards in Spanish preschool children: the PREFIT project. Pediatr Res 2020;87:501-10.

42 Schröder H, Ribas L, Koebnick C, et al. Prevalence of abdominal obesity in Spanish children and adolescents. do we need waist circumference measurements in pediatric practice? PLoS One 2014;9:e87549. 